\title{
Clinical Outcomes after Unplanned Extubation in a Surgical Intensive Care Population: Reply
}

\author{
Hyun-Kyu Yoon · Han-Seul Park · Hee-Pyoung Park
}

Published online: 3 April 2014

(C) Société Internationale de Chirurgie 2014

To the Editor,

We appreciate that Coskun et al. [1] showed great enthusiasm for our paper [2]. In principle, we agree with their opinion that difficult intubation and increased risk of pulmonary complications are associated with obesity. They suggest that body mass index might be a risk factor of unplanned extubation. However, body mass index was not associated with unplanned extubation in our study. Body mass index was $23.9 \pm 3.8$ in patients without unplanned extubation and $24.2 \pm 3.4 \mathrm{~kg} / \mathrm{m}^{2}$ in those with unplanned extubation $(P=0.536)$. The incidence of morbid obesity (body mass index $>30 \mathrm{~kg} / \mathrm{m}^{2}$ ) was not different between the two groups. Moreover, body mass index was not statistically different between reintubated and non-reintubated patients after unplanned extubation $(P=0.752)$.

With respect to APACHE IV score, we agree with their opinion that it is very useful for predicting in-hospital mortality and it is a good method for evaluating patients with prolonged ICU admission. In particular, the APACHE IV model includes 116 detailed admitting diagnostic options so that the model promotes outcome analysis in specific subgroups. Conversely, the large number of variables requires a relatively longer time for data abstraction. Thus, APACHE II score is still in use worldwide because of its simplicity and easy accessibility.

The APACHE IV model shows good discrimination and calibration in predicting in-hospital mortality in ICU patients in the US, where the model was developed [3].

\footnotetext{
H.-K. Yoon · H.-S. Park · H.-P. Park ( $₫)$

Department of Anesthesiology and Pain Medicine, Seoul National University College of Medicine, Seoul National University Hospital, 103 Daehangno, Jongno-gu, Seoul 110-744, Korea

e-mail: hppark@snu.ac.kr
}

However, a recent study showed that, outside of the US, the discrimination performance of the APACHE IV model is good but its calibration performance is poor [4]. According to our unpublished data, the APACHE IV model revealed good discrimination (area under the receiver operating characteristic curve, $\mathrm{AUC}=0.80$ ) but poor calibration (Hosmer and Lemeshow $C$-statistic test $=220.33$, $P<0.001)$ for predicting in-hospital mortality in a Korean surgical ICU. The overall discrimination and calibration capabilities of APACHE IV were similar to those of APACHE II (AUC $=0.85$; Hosmer and Lemeshow $C$ statistic test $=560.94, P<0.001)$. Therefore, we think that there is no significant difference in predicting in-hospital mortality in our study populations between the two APACHE models.

Conflict of interest None.

\section{References}

1. Coskun AK, Mentes O, Harlak A (2014) Comment on: clinical outcomes after unplanned extubation in a surgical intensive care population. World J Surg. doi:10.1007/s00268-014-2471-9

2. Lee JH, Lee HC, Jeon YT et al (2014) Clinical outcomes after unplanned extubation in a surgical intensive care population. World J Surg 38:203-210. doi:10.1007/s00268-013-2249-5

3. Zimmerman JE, Kramer AA, McNair DS, Malila FM (2006) Acute Physiology and Chronic Health Evaluation (APACHE) IV: hospital mortality assessment for today's critically ill patients. Crit Care Med 34:1297-1310

4. Brinkman S, Bakhshi-Raiez F, Abu-Hanna A et al (2011) External validation of Acute Physiology and Chronic Health Evaluation IV in Dutch intensive care units and comparison with Acute Physiology and Chronic Health Evaluation II and Simplified Acute Physiology Score II. J Crit Care 26(1):105.e11-105.e18 